\title{
Orthogonal Chirp-Division Multiplexing for Performance Enhanced Optical/Millimeter-Wave 5G/6G Communications
}

\author{
Colm Browning ${ }^{1}$, Xing Ouyang ${ }^{2}$, Devika Dass ${ }^{1}$, Giuseppe Talli ${ }^{2}$ and Paul Townsend ${ }^{2}$ \\ 1. School of Electronic Engineering, Dublin City University, Dublin D09 W6Y4, Ireland \\ 2. Photonic Systems Group, Tyndall National Institute, Cork T12 R5CP, Ireland \\ Authore-mail address: colm.browning@dcu.ie
}

\begin{abstract}
Orthogonal chirp-division multiplexing is deployed as a novel waveform in an optical/millimeter-wave system. Enhanced channel estimation gives a 5-dB receiver sensitivity improvement over a conventional OFDM implementation, and compatibility with 256-QAM at $60-\mathrm{GHz}$ is experimentally demonstrated.

OCIS codes: (060.4510) Optical Communications; (060.2840) Heterodyne; (060.4080) Modulation.
\end{abstract}

\section{Introduction}

Focus is now shifting toward $6 \mathrm{G}$ communications, driven by the ever-increasing global mobile data traffic, which is projected to reach over two zettabytes in 2025. 6G key performance indicators (KPIs) of data rate scaling, reduced latency and enhanced security are likely to be delivered through a 'super-convergence' of optical and wireless systems/technologies, and further leveraging of the millimeter-wave (mm-wave) and terahertz $(\mathrm{THz})$ frequency bands for mobile and high-throughput applications [1]. The continued trend toward network centralization and the proliferation of optical access infrastructure means that fiber networks will be pivotal to the wide-scale delivery of future mobile services. However, currently deployed binary digital radio-over-fiber (D-RoF) technologies inhibit network scaling due to poor spectral efficiency [2]. To tackle this challenge an analog (A)-RoF approach can be taken where the mobile signal is transmitted through the fiber link in its native multi-carrier modulation (MCM) format; thereby exploiting the signal's high spectral efficiency, and reducing system complexity through the avoidance of the digitization process at the remotely deployed radio head (RRH). Hybrid optical-wireless systems can also be of great benefit to the mass deployment of $\mathrm{mm}$-wave/ $\mathrm{THz}$ communications. By utilizing an optical heterodyne approach - whereby two optical carriers are simultaneously detected at the RRH by a photo-diode (PD), producing an electrical carrier at the frequency difference between the optical carriers - such hybrid systems offer several advantages over all-electronic mm-wave generation approaches, including: (i) reduction in RRH cost and complexity, (ii) inherent compatibility with optical distribution networks, and (iii), potential for wide electrical carrier flexibility/inter-operation enabled by optical source tuning. Indeed, the authors' previous works have shown that hybrid optical-wireless systems are capable of supporting A-RoF transmission, provided that the performance limiting laser phase noise can be successfully mitigated $[3,4]$. To further tackle the challenges posed by transmission in $\mathrm{mm}-w a v e / \mathrm{THz}$ environments, a departure from the ubiquitous orthogonal frequency division multiplexing (OFDM) technologies, which have sustained into the 5G era, is expected [1]. Previously, we have proposed orthogonal chirp-division multiplexing (OCDM) as a novel waveform which, compared to OFDM, offers increased robustness to the severe multi-path impairments due its unique chirp spread-spectrum form [5]. In addition, OCDM's discrete implementation readily facilitates superior receiver channel estimation (CE) and equalization (EQ) [6]. Because of these advantages, recent works have assessed OCDM's performance favorably in both optical transport [6] and wireless [7] systems. In [8], the authors highlight OCDM's tolerance to wireless mm-wave interference in an A-RoF system which includes a photo-generated mm-wave signal. In this work, we demonstrate performance improvement of $5 \mathrm{G}$ NR compatible, and $6 \mathrm{G}$ envisioned, signals by utilizing OCDM with pulse compression (PC) based $\mathrm{CE}$ to alleviate amplitude noise effects in a hybrid optical $/ \mathrm{mm}$-wave A-RoF system operating at $60 \mathrm{GHz}$. When used in combination with phase noise mitigation in the optical domain, this approach allows the enhancement of centralized A-RoF transmission over $10 \mathrm{~km}$ of fiber; with bit error ratio (BER) results showing that OCDM with PC CE exhibits a $5 \mathrm{~dB}$ improvement in receiver sensitivity over equivalent OFDM signals demodulated using a direct frequency domain (FD) CE. Ultimately, this enables the transmission of a $200 \mathrm{MHz} 256-$ QAM OCDM signal (roughly in-line with 5G NR numerology [9]) with received error vector magnitudes (EVM) as low as 3.3\%, and an expanded $1 \mathrm{GHz} 64-\mathrm{QAM}$ OCDM signal delivering a raw data rate of $6 \mathrm{~Gb} / \mathrm{s}$.

\section{Orthogonal Chip-Division Multiplexing and Enhanced Channel Estimation}

Our proposed OCDM waveform is based on the principle of orthogonally multiplexing a set of chirped waveforms of the same bandwidth. The spread-spectrum-like features of this waveform offer robustness to multipath fading effects and in [5] we have shown how it can outperform OFDM in a wireless channel. OCDM's (inverse) discrete Fresnel transform ((I)DFnT) - required to achieve orthogonality in the chirp domain - can be synthesized utilizing the (I)DFT as a basis; offering ease of integration with existing OFDM digital signal processing (DSP). In [6], we 


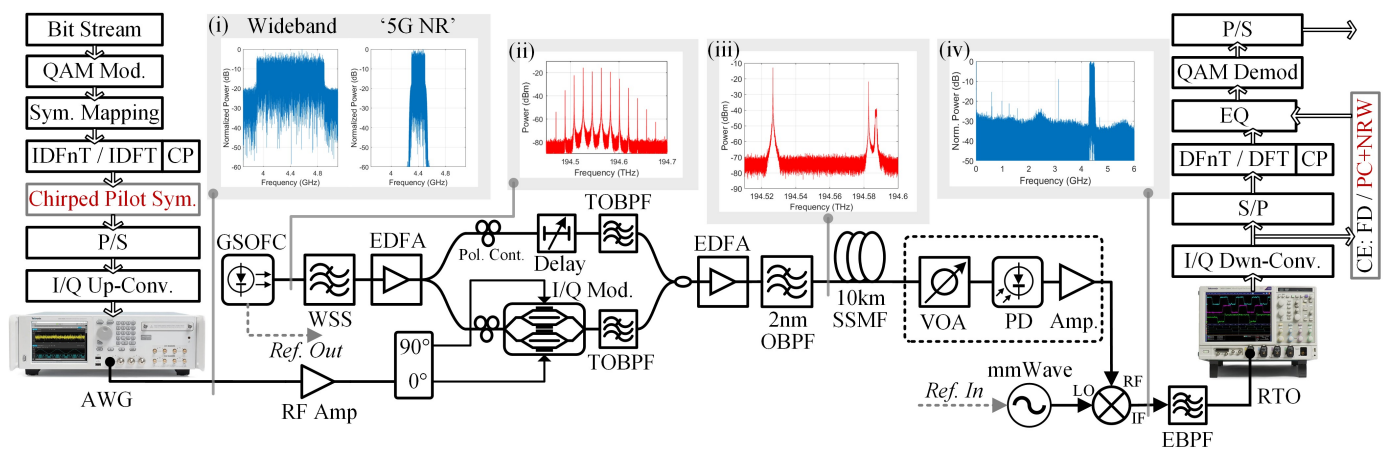

Fig. 1. Experimental optical/mm-wave system setup with electrical and optical spectra along the path shown in (i), (ii), (iii) and (iv).

proposed the use of a chirped signal, obtained directly from OCDM's DFnT, as a pilot symbol for CE. By exploiting the PC properties of this chirped pilot, the channel state information (CSI) can be estimated at the receiver. For improved estimator accuracy, a noise rejection window (NRW) is implemented after PC to remove excess noise. The resultant estimator is unbiased and to converges on the actual channel CSI. The PC+NRW CE provides the same performance as a minimum mean squared error (MMSE) estimator with reduced computational complexity [6], and requires one additional DFnT (in addition to the NRW function) compared to the FD CE used in this work.

\section{Experimental Setup: OCDM-based mmWave A-RoF Transmission}

Fig. 1 shows the expeirmental setup used to examine the feasibility of OCDM transmission in a $10 \mathrm{~km}$ centralized $60 \mathrm{GHz}$ hybrid optical/mm-wave A-RoF system. The performances of a 5G NR compatible 256-QAM multicarrier signal, and an expanded bandwidth 64-QAM multicarrier signal, were evaluated. For the 256/64-QAM OFDM signals, a 1024-point IDFT was used to modulate $820 / 512$ subcarriers with a spacing of $244 / 1953 \mathrm{kHz}$, respectively. Exactly the same numerology was implemented for OCDM whose orthogonal chirps were assigned via an IDFnT. The 256 and 64-QAM signals (Fig. 1 (i)) exhibited bandwidths of $200 \mathrm{MHz}$ and $1 \mathrm{GHz}$, and raw data rates of 1.6 and $6 \mathrm{~Gb} / \mathrm{s}$, respectively. A 288-sample cyclic prefix (CP) was inserted and, where applicable, a single chirped pilot symbol (generated by the IDFnT) was inserted to enable PC+NRW CE at the receiver (otherwise, a single OFDM symbol was used for direct FD CE and zero-forcing EQ). After signal generation, the waveforms were digitally upsampled and I/Q mixed to an intermediate frequency (IF) of $4.4 \mathrm{GHz}$. The IF signals were loaded into an arbitrary waveform generator (AWG) operating at $20 \mathrm{GSa} / \mathrm{s}$. The AWG output signal was amplified and used to drive an optical I/Q modulator via an electrical $90^{\circ}$ hybrid - allowing optical single sideband (SSB) modulation. A gainswitched optical frequency comb (GSOFC) source (output spectra in Fig. 1(ii), further technical details in [3]) in combination with a wavelength selective switch (WSS) was used to provide two correlated optical carriers with a $56.1 \mathrm{GHz}$ frequency separation. After amplification of the carriers using an Erbium doped fiber amplifier (EDFA), one carrier was SSB modulated, while the other was passed through a tunable optical delay line to ensure phase noise mitigation in the system [3]. After recombination, the composite signal (Fig 1. (iii)) was again amplified and sent through a $10 \mathrm{~km}$ standard single mode fiber (SSMF) reel. A variable optical attenuator (VOA) was used to control the power falling on a $70 \mathrm{GHz}$ photo-diode (PD). The photo-generated $60.5 \mathrm{GHz}$ OCDM/OFDM signal was

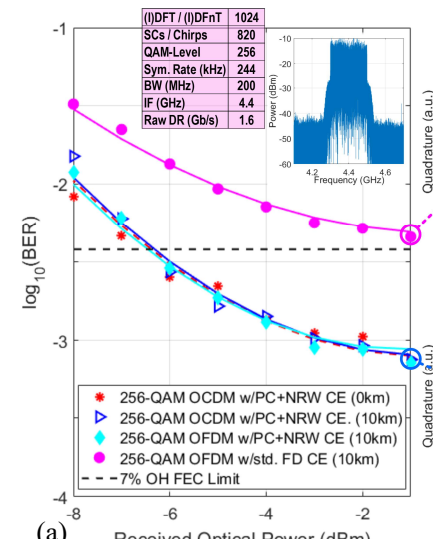

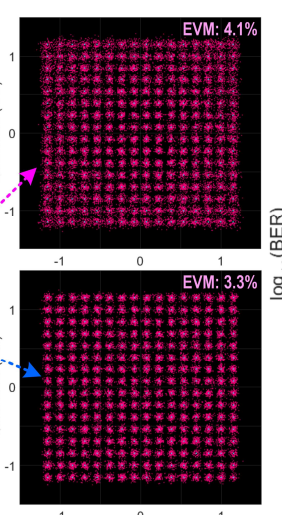

In-Phase (a.u.)

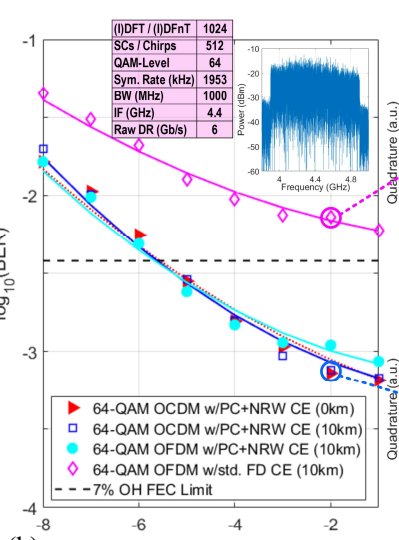

(b) Received Optical Power $(\mathrm{dBm})$

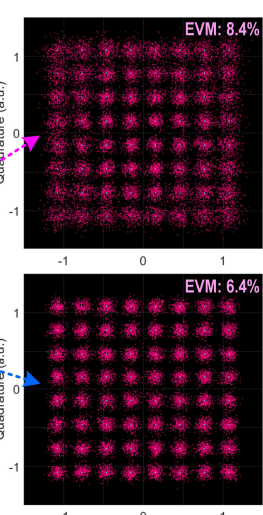

In-Phase (a.u.)

Fig. 2. ROP Vs. BER for 0 and $10 \mathrm{~km}$ with inset rx. constellations, IF spectra and numerology for 256 (a) and 64 (b) QAM OCDM/OFDM signals. 
then mixed down to the IF (Fig. 1(iv)) using a $56.1 \mathrm{GHz}$ local oscillator (LO), band-pass filtered (BPF), and sampled using a real-time oscilloscope (RTS) operating at $100 \mathrm{GSa} / \mathrm{s}$. Wireless transmission was not performed in this experimental work. As well as the DSP blocks outlined in Fig. 1, BER and EVM calculation were performed offline.

\section{Results and Discussion}

Fig. 2 shows the $0 \mathrm{~km}$ and $10 \mathrm{~km}$ transmission performances, in terms of received optical power (ROP) versus BER, of the 256-QAM (a) and 64-QAM (b) OCDM signals EQ'd using the PC+NRW CE. While the PC+NRW CE process is facilitated by a chirped pilot symbol produced through OCDM's IDFnT, it can in theory be applied to any MCM scheme. So, for comparison, the figures show results where the equivalent OFDM signals are EQ'd at the receiver using the FD CE approach, as well as the PC-based CE. Fig. 2 (a) shows how the transmission of 256-QAM OFDM with FD CE exhibits an error floor above the FEC threshold of $3.8 \times 10^{-3}$, due to the presence of amplitude noise in the optical $/ \mathrm{mm}$-wave system arising mainly from a combination of optical source relative intensity noise (RIN) and amplified spontaneous emission (ASE) from the EDFAs. This performance limitation is lifted when the PC+NRW CE is applied to both the OCDM and OFDM signals; achieved by the enhanced noise suppression properties of the estimator. The figure shows a $5 \mathrm{~dB}$ improvement in receiver sensitivity close to the FEC threshold. For this $200 \mathrm{MHz}$ signal, both formats display similar performance when PC+NRW CE is used, with BERs down to $7.5 \times 10^{-4}$. As evidenced by the constellation diagrams in Fig. 2(a), the effects of laser phase noise are eradicated in all cases by the system design, which ensures optical carrier correlation the PD. This combination of phase and amplitude noise mitigation allows the system to support 256-QAM operation with an EVM of just 3.3\%.

Similar trends are exhibited in Fig. 2 (b) which shows transmission performances for the $1 \mathrm{GHz}$ 64-QAM signal. $\mathrm{PC}+\mathrm{NRW} \mathrm{CE}$ once again alleviates the system error floor exhibited by the OFDM signal with FD CE - whose associated constellation diagram at a ROP of $-2 \mathrm{dBm}$ (EVM: $8.5 \%$ ) displays significant distortion of outer constellation points. This effect occurs with IF (i.e. I/Q up-converted) signals in the presence of amplitude noise where QAM symbols requiring a relatively higher SNR display a Gaussian-like noise distribution in both I and Q planes. As the impact of system amplitude noise is diminished by the PC+NRW CE, the equivalent OCDM constellation at $-2 \mathrm{dBm}$ does not display this effect, with EVM reducing to $6.4 \%$ (BER: $7.6 \times 10^{-4}$ ). At higher ROPs OCDM exhibits $\sim 1 \mathrm{~dB}$ improvement in receiver sensitivity compared to OFDM in cases where the PC+NRW CE is used. This is due to OCDM's increased robustness to channel frequency selectivity [6], which is evident for this 1 $\mathrm{GHz}$ bandwidth signal (see Fig. 2(b) inset IF spectrum) due to the frequency response of the mm-wave components used. This highlights the advantages of OCDM for wideband hybrid optical-wireless applications envisioned for 6G.

\section{Conclusion}

An effective convergence of photonic and wireless technologies is required to support mobile network evolution toward cost-effective mm-wave operation. Results presented show that the novel waveform OCDM is compatible with a hybrid optical/mm-wave system which supports A-RoF transmission, flexible remote mm-wave generation and optical phase noise mitigation. Moreover, through the application of OCDM's PC-based estimator, system performance limitations can be overcome by an enhanced suppression of channel noise effects. This results in a $5 \mathrm{~dB}$ improvement in receiver sensitivity compared to OFDM with FD CE, and facilitates 256-QAM operation at $60 \mathrm{GHz}$, as well wideband 64-QAM operation delivering $6 \mathrm{~Gb} / \mathrm{s}$; with each exhibiting BERs well below the FEC limit. Considering OCDM's demonstrated potential in the wireless domain, the proposed system design represents a highly promising synergy of emerging wireless and photonic technologies capable of providing high speed and high frequency mobile communications for future $6 \mathrm{G}$ applications.

\section{Acknowledgements}

This work has emanated from research supported by Science Foundation Ireland under grant numbers 18/SIRG/5579 and 12/RC/2276-P2, and by the TTSI3 Pipeline Fund under grant number SI-2020-3013.

\section{References}

[1] H. Tataria, et al., "6G wireless systems: Vision, requirements, challenges, insights, and opportunities," arXiv: 2008.03213 (2020).

[2] T. Pfeiffer, "Next generation mobile fronthaul and midhaul architectures [Invited]," in IEEE/OSA JOCN 7, B38-B45 (2015).

[3] C. Browning et al., "Gain-switched optical frequency combs for future mobile radio-over-fiber millimeter-wave systems," in IEEE JLT 36, 4602-4610, Oct. $1,2018$.

[4] C. Browning et al., "Phase noise robust optical heterodyne system for reduced complexity millimeter-wave analog radio-over-fibre," in ECOC, Dublin, Ireland, 2019

[5] X. Ouyang, and J. Zhao, “Orthogonal chirp division multiplexing," IEEE Trans. Commun. 64, 3946-3957 (2016).

[6] X. Ouyang, et al., "Robust channel estimation for coherent optical orthogonal chirp-division multiplexing with pulse compression and noise rejection," IEEE JLT 36, 5600-5610, (2018).

[7] R. Bomfin et al., "Performance assessment of orthogonal chirp division multiplexing in MIMO space time coding," in IEEE 5GWF, Dresden, Germany, 2019.

[8] F. Lu et al., "Orthogonal chirp division multiplexing in millimeter-wave fiber-wireless integrated systems for enhanced mobile broadband and ultra-reliable communications," in OFC, Los Angeles, USA, 2017.

[9] 3GPP, "Release 15: TR 21.915," 5.5.4 Radio Physical layer aspects, 25-27 (2019) 